\title{
Timoshenko Beam Theory for the Flexural Analysis of Moderately Thick Beams - Variational Formulation, and Closed Form Solution
}

\author{
Charles Chinwuba Ike \\ Department of Civil Engineering, Enugu State University of Science \& Technology, Enugu State, Nigeria
}

Corresponding Author Email: ikecc2007@yahoo.com

https://doi.org/10.18280/ti-ijes.630105

Received: 22 January 2019

Accepted: 19 March 2019

\section{Keywords:}

Timoshenko beam theory, moderately thick beams, total potential energy functional, Euler-Lagrange differential equations, differential equations of equilibrium, shear deformation

\begin{abstract}
In this study, the Timoshenko first order shear deformation beam theory for the flexural behaviour of moderately thick beams of rectangular cross-section is formulated from vartiational principles, and applied to obtain closed form solutions to the flexural problem of moderately thick rectangular beams. The total potential energy functional for the moderately thick beam flexure problem was formulated by considering the contribution of shearing deformation to the strain energy. Euler-Lagrange conditions were then applied to obtain the system of two coupled ordinary differential equations of equilibrium. The problem of moderately thick beam with simply supported ends subject to uniformly distributed transverse load on the entire span was solved in closed form to obtain the transverse deflection as the sum of the flexural and shear components. Another problem of moderately thick cantilever beam under point load at the free end was solved in closed form to illustrate the solution of the governing equations. The transverse deflection was similarly obtained as the sum of shear and bending components. The bending component of deflection was found to be identical with the Euler-Bernoulli results while the shear component was found to be dependent on the square of the ratio of the beam thickness, $t$, to the span, $l$. It was found that as $t / l<0.02$, the contribution of shear to the overall deflection is insignificant; but becomes significant for $t / l>0.10$. The findings are in excellent agreement with the technical literature.
\end{abstract}

\section{INTRODUCTION}

The classical theory of beam flexure, also called the EulerBernoulli beam theory (EBT) neglects the effects of the transverse shear strains and deformation, and stress concentration [1-5]. The theory is built on the Euler-Bernoulli hypothesis which assumes that the transverse normal to the neutral axis remains normal (orthogonal) to the vertical axis during and after flexural deformation; and this implies that there are no transverse shear stresses. The disregarding of transverse shear deformation in the formulation of the governing equations make EBT suitable for thin (slender) beams, but unsuitable for moderately thick (deep) beams and thick beams where shear deformation plays a significant role in the flexural behaviour [6-8]. EBT thus underestimates the deflections of moderately thick and thick beams where shear deformation effects significantly contribute to their behaviours

Timoshenko [9] presented a first order shear deformation theory for the flexural behaviour of moderately thick and thick beams that accounts for the effects of shear deformation and rotatory inertia. The major drawback of his theory is the assumption of the transverse shear strain distribution to be uniform across the beam cross-section at any point, thus requiring problem dependent shear modification factors to accurately represent the strain energy of deformation.

Cowper [10] presented mathematical expressions for the shear correction/modification factors of beams in terms of the Poisson's ratio and geometrical properties of different crosssectional shapes such as inner and outer radii.
The need to overcome the limitations of the EBT and the Timoshenko first order shear deformation theory motivated research in the formulation of first, second, third order and higher order shear deformation theories for beams by Levinson, Reddy, Mindlin, Vlasov and Leontiev, Stein, Touratier, Shimpi, Ghugal, Sayyad etc. [11-14]. Levinson [15], Krishna Murty [16], Baluch et al. [17], Bhimaraddi and Chandrashekhara [18], Brickford [19] presented parabolic shear deformation theories of beams by assuming a higher variation of axial (longitudinal) displacement field in terms of the thickness coordinate variable, $z$. Their parabolic shear deformation beam theories were formulated to apriori satisfy the shear stress free boundary conditions of the top and bottom surfaces of the beam, and thus remove the necessity of shear correction/modification factor. Trigonometric shear deformation theories of beams were formulated and developed by Touratier [20], Vlasov and Leontiev [21], Stein [22] for thick beams. Their formulations however violated the shear stress free boundary conditions at the top and bottom surfaces of the beam. Ghugal and Kapdis et al. [23] and Ghugal [24] improved the earlier formulations of trigonometric shear deformation beam theories by presenting shear deformation beam theories that satisfy the shear stress free boundary conditions at the top and bottom surfaces of the beam.

In the present study, the Timoshenko first order shear deformation beam theory is derived from fundamental principles, using the method of variational calculus. The governing system of two ordinary differential equations of equilibrium obtained are then solved in closed form for the 
specific cases of moderately thick rectangular beams, subject to uniformly distributed transverse load and moderately thick rectangular cantilever beam with a point load at the free end.

\section{Research aim and objectives}

The general aim of this study is to present a general formulation of the Timoshenko beam theory and then apply it to the flexural analysis of moderately thick beams. The specific objectives include:

(i) to formulate the governing partial differential equations of equilibrium of Timoshenko beams using variational calculus methods.

(ii) to solve the governing system of ordinary differential equations of the Timoshenko beam for the specific cases of: (a) simply supported moderately thick beams subject to uniformly distributed transverse load $p_{0}$ over the entire beam span; (b) moderately thick cantilever beams subject to point load $P$ at the free end.

\section{THEORETICAL FRAMEWORK}

The study considered a thick beam of width $b$, thickness, $t$ and span $l$ with a longitudinal axis coincident with the $x$ coordinate direction. The origin of the three dimentional (3D) Cartesian coordinate axis used is defined at the left end of the beam. The beam thus occupies the $3 \mathrm{D}$ region defined as: $0 \leq$ $x \leq l,-\frac{b}{2} \leq y \leq \frac{b}{2},-\frac{t}{2} \leq z \leq \frac{t}{2}$

\section{Variational formulation of the Timoshenko beam theory}

The assumptions of the formulation are:

(i) The longitudinal axis of the unloaded undeformed beam is straight.

(ii) All loads applied to the beam act transverse to the longitudinal axis.

(iii) Line elements that are normal to the middle line of the beam in the undeformed configuration will deform only in the vertical direction, and will also remain vertical during deformation. Plane cross-sections of the beam which are initially orthogonal to the longitudinal axis will remain plane after deformation.

(iv) Line elements that are tangential to the middle line (center line) will undergo a rotation $\varphi(x)$ which would correspond to the shear angle $\gamma_{x z}$ at an arbitrary point along the centerline.

(v) The total slope of the centerline results from the effects of bending deformation and shear deformation and can be expressed as the sum of the rotations due to shear deformation $\varphi(x)$ and the rotation due to bending deformation $\alpha(x)$. Thus

$$
\frac{\partial w}{\partial x}=\alpha(x)+\varphi(x)
$$

(vi) The transverse shear strain $\gamma_{x z}$ is constant at all points over a given cross-section of the beam. This implies the shear stress distribution is constant or uniform over the cross-section at any point on the longitudinal axis. A constant distribution of shear stress over the crosssection at any point on the longitudinal axis for applied transverse load distribution on the upper surface $z=-\frac{t}{2}$, and no traction on the lower surface $z=\frac{t}{2}$ violates the theory of elasticity solutions of the problem. The assumption of constant transverse shear strain is however used to minimize computational and analytical rigours and any resulting errors introduced are accounted for by the introduction of shear correction factors, $k$.

(vii) The beam material is linear elastic, homogeneous and isotropic. Hence, the generalised Hooke's stress-strain laws are valid. Thus:

$$
\begin{aligned}
& \varepsilon_{x x}=\frac{1}{E}\left(\sigma_{x x}-\mu \sigma_{y y}-\mu \sigma_{z z}\right) \\
& \varepsilon_{y y}=\frac{1}{E}\left(\sigma_{y y}-\mu \sigma_{x x}-\mu \sigma_{z z}\right) \\
& \varepsilon_{z z}=\frac{1}{E}\left(\sigma_{z z}-\mu \sigma_{x x}-\mu \sigma_{y y}\right) \\
& \gamma_{x y}=\frac{\tau_{x y}}{G} \\
& \gamma_{y z}=\frac{\tau_{y z}}{G} \\
& \gamma_{z x}=\frac{\tau_{z x}}{G}
\end{aligned}
$$

where $\sigma_{x x}, \sigma_{y y}, \sigma_{z z}$ are normal stresses $\tau_{x z}, \tau_{y z}$ and $\tau_{x y}$ are shear stresses, $\varepsilon_{x x}, \varepsilon_{y y}, \varepsilon_{z z}$ are normal strains, while $\gamma_{x z}, \gamma_{y z}$ and $\gamma_{x y}$ are shear strains, $E$ is the Young's modulus of elasticity, $G$ is the shear modulus, $\mu$ is the Poisson's ratio.

$G=\frac{E}{2(1+\mu)}$

(vii) The deformations and strains are considered so small, and the strain-displacement equations of infinitesimal elasticity are used.

Thus, the kinematic equations are:

$$
\begin{aligned}
& \varepsilon_{x x}=\frac{\partial u}{\partial x} \\
& \varepsilon_{y y}=\frac{\partial v}{\partial y} \\
& \varepsilon_{z z}=\frac{\partial w}{\partial z} \\
& \gamma_{x y}=\frac{\partial u}{\partial y}+\frac{\partial v}{\partial x} \\
& \gamma_{x z}=\frac{\partial u}{\partial z}+\frac{\partial w}{\partial x} \\
& \gamma_{y z}=\frac{\partial v}{\partial z}+\frac{\partial w}{\partial y}
\end{aligned}
$$

\section{Displacement field}

The displacement field components about the $x, y$, and $z$ 
coordinate axis given respectively by $u(x, y, z), v(x, y, z)$ and $w(x, y, z)$ are given by:

$u(x, y, z)=-z \alpha(x)=-z\left(\frac{d w}{d x}-\varphi(x)\right)$

$v(x, y, z)=0$

$w(x, y, z)=w(x, y=0, z=0)=w(x)$

It is noted that the displacement component in the longitudinal direction ( $x$-coordinate) is caused solely by bending deformation.

\section{Strain fields}

Applying the strain-displacement relations, the strain fields are obtained as:

$\varepsilon_{x x}=\frac{\partial}{\partial x}\left(-z\left(\frac{d w}{d x}-\varphi(x)\right)\right)=\frac{\partial}{\partial x}(-z \alpha(x))$

$\varepsilon_{x x}=-z\left(\frac{d^{2} w}{d x^{2}}-\frac{d \varphi}{d x}\right)=-z \frac{d \alpha(x)}{d x}$

$\varepsilon_{x x}=-z\left(w^{\prime \prime}(x)-\varphi^{\prime}(x)\right)$

$\varepsilon_{y y}=\frac{\partial v}{\partial y}=0$

$\varepsilon_{z z}=\frac{\partial w}{\partial z}=0$

$\gamma_{x y}=\frac{\partial u}{\partial y}+\frac{\partial v}{\partial x}=0$

$\gamma_{y z}=\frac{\partial v}{\partial z}+\frac{\partial w}{\partial y}=0$

$\gamma_{x z}=\frac{\partial}{\partial z}\left(-z\left(\frac{d w}{d x}-\varphi(x)\right)\right)+\frac{\partial w}{\partial x}$

$\gamma_{x z}=-\left(\frac{d w}{d x}-\varphi(x)\right)+\frac{\partial w}{\partial x}=\varphi(x)$

\section{Stress fields}

The stress fields are obtained from the stress-strain laws as:

$\sigma_{x x}=E \varepsilon_{x x}=-E z\left(\frac{d^{2} w}{d x^{2}}-\frac{d \varphi}{d x}\right)=-E z \frac{d \alpha(x)}{d x}$

$\sigma_{y y}=0$

$\sigma_{z z}=0$

$\tau_{x z}=G \gamma_{x z}=G \varphi(x)$

$$
\tau_{y z}=0
$$

$$
\tau_{x y}=0
$$

Introduction of the shear correction (modification) factor, $k$ yields:

$\tau_{x z}^{c}=k G \varphi(x)$

where the superscript $c$ refers to corrected (modified) shear stress distribution.

\section{Internal stress resultants}

$$
\begin{aligned}
& M=\int_{-b / 2}^{b / 2} \int_{-t / 2}^{t / 2} \sigma_{x x} z d z d y \\
& M=\int_{-b / 2}^{b / 2} d y \int_{-t / 2}^{t / 2} \sigma_{x x} z d z=\int_{-t / 2}^{t / 2} \sigma_{x x} b z d z \\
& M=\int_{-t / 2}^{t / 2}-E \frac{d \alpha}{d x} b z^{2} d z
\end{aligned}
$$

$M=-E \int_{-t / 2}^{t / 2} b z^{2} d z \frac{d \alpha}{d x}$

$M=-E\left[\frac{b z^{3}}{3}\right]_{-t / 2}^{t / 2} \frac{d \alpha}{d x}=-E I \frac{d \alpha}{d x}$

$Q(x)=\int_{-b / 2}^{b / 2} \int_{-t / 2}^{t / 2} \tau_{x z} d z d y$

$Q=\int_{-b / 2}^{b / 2} d y \int_{-t / 2}^{t / 2} \tau_{x z} d z=b \int_{-t / 2}^{t / 2} d z \tau_{x z}$

$Q=b t \tau_{x z}=A \tau_{x z}=G A \varphi(x)$

$Q^{T}(x)=k Q=k G A \varphi(x)$

\section{Total potential energy functional $\Pi$}

The total potential energy functional $\Pi$ for the moderately thick beam is given by the sum of the strain energy $U$ and the potential of the external load $V$ as:

$$
\begin{aligned}
& \Pi=U+V \\
& \begin{aligned}
U=\frac{1}{2} \iiint_{R^{3}}\left(\sigma_{x x} \varepsilon_{x x}\right. & +\sigma_{y y} \varepsilon_{y y}+\sigma_{z z} \varepsilon_{z z} \\
& \left.+\tau_{x y} \gamma_{x y}+\tau_{y z} \gamma_{y z}+\tau_{x z} \gamma_{x z}\right) d x d y d z
\end{aligned}
\end{aligned}
$$


where $R^{3}$ is $0 \leq x \leq l,-b / 2 \leq y \leq b / 2, \quad-t / 2 \leq z \leq t / 2$

$V=-\int_{0}^{l} p(x) w(x) d x$

$U=\frac{1}{2} \int_{-t / 2}^{t / 2} \int_{-b / 2}^{b / 2} \int_{0}^{l}\left(\sigma_{x x} \varepsilon_{x x}+\tau_{x z} \gamma_{x z}\right) d x d y d z$

$U=\frac{1}{2} \int_{-t / 2-b / 2}^{t / 2} \int_{0}^{b / 2} \int_{0}^{l}\left(E \varepsilon_{x x}^{2}+k G \gamma_{x z}^{2}\right) d x d y d z$

$U=\frac{1}{2} \iiint_{R^{3}} E \varepsilon_{x x}^{2} d x d y d z+\frac{1}{2} \iiint_{R^{3}} k G \gamma_{x z}^{2} d x d y d z$

$U=\frac{1}{2} \int_{-b / 2}^{b / 2} d y \int_{-t / 2}^{t / 2} \int_{0}^{l} E\left(-z \frac{d \alpha}{d x}\right)^{2} d x d z$

$+\frac{1}{2} k \int_{-b / 2}^{b / 2} d y \int_{-t / 2}^{t / 2} \int_{0}^{l} G \cdot(\varphi(x))^{2} d x d z=U_{b}+U_{s}$

$U_{b}=\frac{1}{2} b E \int_{-t / 2}^{t / 2} z^{2} d z \int_{0}^{l}\left(\frac{d \alpha}{d x}\right)^{2} d x$

$U_{b}=\frac{1}{2} E I \int_{0}^{l}\left(\frac{d \alpha}{d x}\right)^{2} d x$

$U_{s}=\frac{1}{2} k b G \int_{-t / 2}^{t / 2} d z \int_{0}^{l}(\varphi(x))^{2} d x$

$U_{s}=\frac{1}{2} b G t \int_{0}^{l}(\varphi(x))^{2} d x$

$$
\begin{aligned}
\Pi=\frac{1}{2} E I \int_{0}^{l}\left(\frac{d \alpha}{d x}\right)^{2} d x \\
\quad+\frac{1}{2} G A \int_{0}^{l} k(\varphi(x))^{2} d x-\int_{0}^{l} p(x) w(x) d x
\end{aligned}
$$

$$
\begin{aligned}
\Pi=\frac{1}{2} E I \int_{0}^{l}\left(\frac{d \alpha}{d x}\right)^{2} d x \\
\quad+\frac{1}{2} G A \int_{0}^{l} k\left(\frac{d w}{d x}-\alpha(x)\right)^{2} d x-\int_{0}^{l} p(x) w(x) d x
\end{aligned}
$$

$$
\begin{aligned}
\Pi=\int_{0}^{l}\left\{\frac{1}{2} E I\left(\frac{d \alpha}{d x}\right)^{2}\right. \\
\left.\quad+\frac{1}{2} G A k\left(\frac{d w}{d x}-\alpha(x)\right)^{2}-p(x) w(x)\right\} d x
\end{aligned}
$$

$$
\Pi=\int_{0}^{l} F\left(x, w(x), \alpha(x), w^{\prime}(x)\right) d x
$$

$$
\begin{aligned}
F\left(x, w(x), \alpha(x), w^{\prime}(x)\right) & =\frac{1}{2} E I\left(\alpha^{\prime}(x)\right)^{2} \\
+ & \frac{1}{2} G A k\left(w^{\prime}(x)-\alpha(x)\right)^{2}-p(x) w(x)
\end{aligned}
$$

The integrand $F$ is seen to be a function of two unknown functions, $\alpha(x)$ and $w(x)$.

$$
\begin{gathered}
F=\frac{1}{2} E I\left(\alpha^{\prime}(x)\right)^{2}+\frac{G A k}{2}\left(\left(w^{\prime}(x)\right)^{2}-2 w^{\prime}(x) \alpha(x)\right. \\
\left.+(\alpha(x))^{2}\right)-p(x) w(x)
\end{gathered}
$$

\section{Differential Equations of Equilibrium}

The differential equations of equilibrium are the equations corresponding to the minimization of the total potential energy functional $\Pi$ and are obtained by applying the Euler-Lagrange conditions for the extremum of $\Pi$. Thus, the Euler-Lagrange conditions are the system of differential equations:

$\frac{\partial F}{\partial w}-\frac{d}{d x}\left(\frac{\partial F}{\partial w^{\prime}}\right)=0$

$\frac{\partial F}{\partial \alpha}-\frac{d}{d x}\left(\frac{\partial F}{\partial \alpha^{\prime}}\right)=0$

$\frac{\partial F}{\partial \alpha}=\frac{G A k}{2}\left(2 \alpha(x)-2 w^{\prime}(x)\right)$

$\frac{\partial F}{\partial \alpha}=G A k\left(\alpha(x)-w^{\prime}(x)\right)$

$\frac{\partial F}{\partial \alpha^{\prime}}=\frac{1}{2} E I \cdot 2 \alpha^{\prime}(x)=E I \alpha^{\prime}(x)$

$\frac{\partial F}{\partial w}=-p(x)$

$\frac{\partial F}{\partial w^{\prime}}=\frac{G A k}{2}\left(2 w^{\prime}(x)-2 \alpha(x)\right)=G A k\left(w^{\prime}(x)-\alpha(x)\right)$

Thus,

$$
\begin{aligned}
& G A k\left(\alpha(x)-w^{\prime}(x)\right)-\frac{d}{d x} E I \alpha^{\prime}(x)=0 \\
& G A k\left(\alpha(x)-w^{\prime}(x)\right)-E I \alpha^{\prime \prime}(x)=0 \\
& -G A k\left(w^{\prime}(x)-\alpha(x)\right)-E I \alpha^{\prime \prime}(x)=0 \\
& E I \frac{d^{2} \alpha}{d x^{2}}+G A k\left(\frac{d w}{d x}-\alpha(x)\right)=0
\end{aligned}
$$


$-p(x)-\frac{d}{d x} G A k\left(w^{\prime}(x)-\alpha(x)\right)=0$

$-p(x)-G A k \frac{d}{d x}\left(\frac{d w}{d x}-\alpha(x)\right)=0$

$G A k\left(\frac{d}{d x}\left(\frac{d w}{d x}-\alpha(x)\right)\right)+p(x)=0$

or,

$G A k \frac{d}{d x}\left(\alpha(x)-\frac{d w}{d x}\right)=p$

$G A k\left(\frac{d \alpha}{d x}-\frac{d^{2} w}{d x^{2}}\right)=p$

\section{Decoupling the differential equations of equilibrium}

From,

$\frac{d M}{d x}=Q(x)$

$\frac{d Q(x)}{d x}=-p(x)$

Thus,

$\frac{d}{d x}\left(-E I \frac{d \alpha}{d x}\right)=Q(x)=-E I \frac{d^{2} \alpha}{d x^{2}}$

Differentiating again,

$\frac{d}{d x}\left(-E I \frac{d^{2} \alpha}{d x^{2}}\right)=-p(x)$

$-E I \frac{d^{3} \alpha}{d x^{3}}=-p(x)$

$E I \frac{d^{3} \alpha}{d x^{3}}=p(x)$

Differentiating Equation (72) twice, we have

$k G A \frac{d^{2}}{d x^{2}}\left(\frac{d \alpha}{d x}-\frac{d^{2} w}{d x^{2}}\right)=\frac{d^{2} p}{d x^{2}}$

$k G A\left(\frac{d^{3} \alpha}{d x^{3}}-\frac{d^{4} w}{d x^{4}}\right)=\frac{d^{2} p}{d x^{2}}$

$k G A\left(\frac{p(x)}{E I}-\frac{d^{4} w}{d x^{4}}\right)=\frac{d^{2} p}{d x^{2}}$

Multiplying by $E I$, $k G A\left(p(x)-E I \frac{d^{4} w}{d x^{4}}\right)=E I \frac{d^{2} p}{d x^{2}}$

$p(x)-E I\left(\frac{d^{4} w}{d x^{4}}\right)=\frac{E I}{k G A} \frac{d^{2} p}{d x^{2}}$

$E I \frac{d^{4} w}{d x^{4}}=p(x)-\frac{E I}{k G A} \frac{d^{2} p}{d x^{2}}$

For the case of simply supported Timoshenko beam subject to a uniformly distributed transverse load of intensity $p_{0}$, the differential equations of equilibrium simplify to the following system of two equations in $\alpha(x)$ and $w(x)$ :

$E I \frac{d^{3} \alpha(x)}{d x^{3}}=p_{0}$

$E I \frac{d^{4} w}{d x^{4}}=p_{0}-\frac{E I}{k G A} \frac{d^{2} p_{0}}{d x^{2}}=p_{0}$

\section{Summary}

The equations of Timoshenko beam theory are:

$\frac{d Q(x)}{d x}=Q^{\prime}(x)=w(x)$

$\frac{d M(x)}{d x}=M^{\prime}(x)=Q(x)$

$\frac{d \alpha(x)}{d x}=\alpha^{\prime}(x)=\frac{M(x)}{E I}$

$\frac{d u(x)}{d x}=u^{\prime}(x)=\alpha(x)-\frac{Q(x)}{k G A}$

\section{RESULTS}

\section{Illustrative Problem}

The work considered as a specific application, a moderately thick beam simply supported at the ends $x=0$ and $x=l$ modelled as a Timoshenko beam, carrying a uniformly distributed transverse load of intensity $p_{0}$ over the entire span as shown in Figure 1.

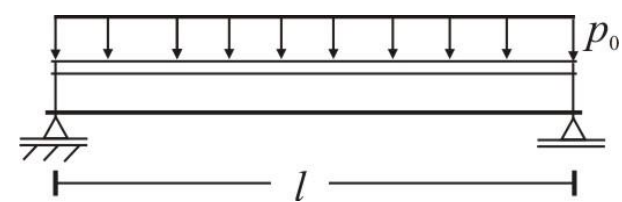

Figure 1. Moderately thick rectangular beam under uniformly distributed transverse load

The boundary conditions are:

$M(x=0)=0$ 
or, $\frac{d \alpha}{d x}(x=0)=0$

$M(x=l)=0$

or, $\frac{d \alpha}{d x}(x=l)=0$

The origin is chosen at the center of the simply supported beam to take advantage of the symmetrical nature of the beam and the loading. The boundary conditions become:

$M(x=l / 2)=0$

or, $\frac{d \alpha}{d x}(x=l / 2)=0$

$M(x=-l / 2)=0$

or, $\frac{d \alpha}{d x}(x=-l / 2)=0$

Integration of Equation (85) yields:

$E I \frac{d^{2} \alpha}{d x^{2}}=p_{0} x+c_{1}$

Integration of Equation (90) yields:

$E I\left(\frac{d \alpha}{d x}\right)=\frac{p_{0} x^{2}}{2}+c_{1} x+c_{2}$

Integration of Equation (100) yields:

$E I \alpha(x)=p_{0} \frac{x^{2}}{6}+c_{1} \frac{x^{2}}{2}+c_{2} x+c_{3}$

where $c_{1}, c_{2}$, and $c_{3}$ are constants of integration.

The symmetrical nature of the problem demands (requires) that $\alpha(x)$ should be an odd function. Thus

$\alpha(-x)=-\alpha(x)$

$c_{1}=0$

$c_{3}=0$

$\frac{d \alpha}{d x}=\frac{1}{E I}\left(\frac{p_{0} x^{2}}{2}+c_{2}\right)$
For $\frac{d \alpha}{d x}(x= \pm l / 2)=\frac{1}{E I}\left(\frac{p_{0}}{2}\left(\frac{l}{2}\right)^{2}+c_{2}\right)=0$

$\frac{p_{0} l^{2}}{8}+c_{2}=0$

$c_{2}=-\frac{p_{0} l^{2}}{8}$

Hence

$\alpha(x)=\frac{1}{E I}\left(\frac{p_{0} x^{3}}{6}-\frac{p_{0} l^{2}}{8} x\right)$

Then

$G A k \frac{d}{d x}\left(\alpha(x)-\frac{d w}{d x}\right)=p(x)=p_{0}$

Integration yields

$G A k\left(\alpha(x)-\frac{d w}{d x}\right)=p_{0} x$

$\alpha(x)-\frac{d w}{d x}=\frac{p_{0} x}{k G A}$

$\frac{d w}{d x}=\alpha(x)-\frac{p_{0} x}{k G A}$

$\frac{d w}{d x}=\frac{1}{E I}\left(\frac{p_{0} x^{3}}{6}-\frac{p_{0} l^{2} x}{8}\right)-\frac{p_{0} x}{k G A}$

Integration yields

$$
\begin{aligned}
w(x)= & \int \frac{d w}{d x} d x \\
& =\int\left\{\frac{1}{E I}\left(\frac{p_{0} x^{3}}{6}-\frac{p_{0} l^{2} x}{8}\right)-\frac{p_{0} x}{k G A}\right\} d x
\end{aligned}
$$

$w(x)=\frac{1}{E I}\left(\frac{p_{0} x^{4}}{24}-\frac{p_{0} l^{2} x^{2}}{16}\right)-\frac{p_{0} x^{2}}{2 k G A}+c_{4}$

Applying the boundary conditions,

$w(x=l / 2)=0$

$w(x=-l / 2)=0$

We have,

$$
\begin{aligned}
w(x=l / 2)=0= & \frac{1}{E I}\left(\frac{p_{0}}{24}\left(\frac{l}{2}\right)^{4}-\frac{p_{0} l^{2}}{16}\left(\frac{l}{2}\right)^{2}\right) \\
& -\frac{p_{0}}{2 k G A}\left(\frac{l}{2}\right)^{2}+c_{4}
\end{aligned}
$$


$\frac{1}{E I}\left(\frac{p_{0} l^{4}}{384}-\frac{p_{0} l^{4}}{64}\right)-\frac{p_{0} l^{2}}{8 k G A}+c_{4}=0$

$c_{4}=\frac{p_{0} l^{2}}{8 k G A}-\frac{1}{E I}\left(\frac{-5 p_{0} l^{4}}{384}\right)$

$c_{4}=\frac{p_{0} l^{2}}{8 k G A}+\frac{5 p_{0} l^{4}}{384 E I}$

Hence,

$$
\begin{aligned}
& w(x)=\frac{p_{0}}{E I}\left(\frac{x^{4}}{24}-\frac{l^{2} x^{2}}{16}\right)-\frac{p_{0} x^{2}}{2 k G A}+\frac{p_{0} l^{2}}{8 k G A}+\frac{5 p_{0} l^{4}}{384 E I} \\
& w(x)=\frac{p_{0}}{E I}\left(\frac{x^{4}}{24}-\frac{l^{2} x^{2}}{16}+\frac{5 l^{4}}{384}\right)+\frac{p_{0}}{2 k G A}\left(\frac{l^{2}}{4}-x^{2}\right) \\
& w(x)=\frac{p_{0}}{E I}\left(\frac{x^{4}}{24}-\frac{l^{2} x^{2}}{16}+\frac{5 l^{4}}{384}\right)+\frac{p_{0}}{2 k b t}\left(\frac{\left(l^{2} / 4\right)-x^{2}}{E / 2(1+\mu)}\right) \\
& w(x)=\frac{p_{0} l^{4}}{24 E I}\left[\left(\frac{x}{l}\right)^{4}-\frac{3}{2}\left(\frac{x}{l}\right)^{2}+\frac{5}{16}\right] \\
& -\frac{p_{0} l^{2}\left((x / l)^{2}-1 / 4\right)}{2 k[E / 2(1+\mu)] b t} \\
& w(x)=\frac{p_{0} l^{4}}{24 E I}\left\{\left[\left(\frac{x}{l}\right)^{4}-\frac{3}{2}\left(\frac{x}{l}\right)^{2}+\frac{5}{16}\right]\right. \\
& \left.-\left(\frac{t}{l}\right)^{2} \frac{2(1+\mu)}{k}\left(\left(\frac{x}{l}\right)^{2}-\frac{1}{4}\right)\right\}
\end{aligned}
$$

$w(x)=w_{f}(x)+w_{s}(x)$

For a rectangular cross-section, $k=5 / 6$ for $\mu=0$.

$k=\frac{10(1+\mu)}{12+11 \mu}$

$k=0.847457627$

$k \simeq \frac{5.08475}{6}$ for $\mu=0.25$

$k=\frac{5.098}{6}$ for $\mu=0.30$

For a circular cross-section,

$k=\frac{6(1+\mu)}{7+6 \mu}$ $k=\frac{1+\mu}{1.305+1.273 \mu}$

For a hollow cylinder of outer radius, $r_{0}$ and inner radius, $r_{i}$,

$k=\frac{6(1+\mu)(1+m)^{2}}{(7+6 \mu)(1+m)^{2}+(20+12 \mu) m^{2}}$

where $m=b / a$

where $a=r_{0}$

and $r_{0}$ is the outer radius, and

$b=r_{i}$

$r_{i}$ is the inner radius.

For a thin-walled tube,

$k=\frac{2(1+\mu)}{4+\mu}$

\section{Maximum deflection}

The maximum deflection occurs at the center, and is:

$w(0)=\frac{p_{0} l^{4}}{24 E I}\left\{\left(\frac{5}{16}\right)+\left(\frac{t}{l}\right)^{2} \frac{2(1+\mu)}{k}\left(\frac{1}{4}\right)\right\}$

$w(0)=\frac{p_{0} l^{4}}{24 E I}\left\{\frac{5}{16}+\left(\frac{t}{l}\right)^{2}\left(\frac{1+\mu}{2 k}\right)\right\}$

For $\mu=0.25$,

$w(0)=\frac{p_{0} l^{4}}{24 E I}\left(\frac{5}{16}+\left(\frac{t}{l}\right)^{2} \frac{1.25}{2(5.08475 / 6)}\right)$

$w(0)=\frac{p_{0} l^{4}}{24 E I}\left(\frac{5}{16}+0.7375\left(\frac{t}{l}\right)^{2}\right)$

$w(0)=\frac{5 p_{0} l^{4}}{384 E I}+3.0729166 \times 10^{-2} \frac{p_{0} l^{4}}{E I}\left(\frac{t}{l}\right)^{2}$

$w^{T}(0)=w_{f}(0)+w_{s}(0)$

$w_{f}(0)=\frac{5 p_{0} l^{4}}{384 E I}$

$w_{s}(0)=3.0721966 \times 10^{-2}\left(\frac{t}{l}\right)^{2} \frac{p_{0} l^{4}}{E I}$

For a semi-circular cross-section 
Table 1. Variation of maximum deflection with ratios of $t / l$ in Timoshenko beams with simply supported ends (case of uniform loads) for $\mu=0.25$

\begin{tabular}{|c|c|c|c|}
\hline$t / l$ & $\begin{array}{l}\times \frac{p_{0} l^{4}}{E I} \\
w_{s}(0)\end{array}$ & $\begin{array}{c}\times \frac{p_{0} l^{4}}{E I} \\
w_{s}(0)\end{array}$ & $\begin{array}{l}\times \frac{p_{0} l^{4}}{E I} \\
w^{T}(0)\end{array}$ \\
\hline 0.005 & $1.302083 \times 10^{-2}$ & $7.68229 \times 10^{-7}$ & $1.30216 \times 10^{-2}$ \\
\hline 0.01 & $1.302083 \times 10^{-2}$ & $3.0729166 \times 10^{-6}$ & $1.30239 \times 10^{-2}$ \\
\hline 0.02 & $1.302083 \times 10^{-2}$ & $1.2291626 \times 10^{-5}$ & $1.3033121 \times 10^{-2}$ \\
\hline 0.05 & $1.302083 \times 10^{-2}$ & $7.68229 \times 10^{-5}$ & $1.3097652 \times 10^{-2}$ \\
\hline 0.08 & $1.302083 \times 10^{-2}$ & $0.01966 \times 10^{-2}$ & $1.32175 \times 10^{-2}$ \\
\hline 0.10 & $1.302083 \times 10^{-2}$ & $3.0729166 \times 10^{-4}$ & $1.332812 \times 10^{-2}$ \\
\hline 0.15 & $1.302083 \times 10^{-2}$ & $0.0691406 \times 10^{-2}$ & $1.3712236 \times 10^{-2}$ \\
\hline 0.20 & $1.302083 \times 10^{-2}$ & $0.12291666 \times 10^{-2}$ & $1.425 \times 10^{-2}$ \\
\hline 0.40 & $1.302083 \times 10^{-2}$ & $0.491666 \times 10^{-2}$ & $1.79375 \times 10^{-2}$ \\
\hline 0.60 & $1.302083 \times 10^{-2}$ & $1.10625 \times 10^{-2}$ & $2.408333 \times 10^{-2}$ \\
\hline 0.80 & $1.302083 \times 10^{-2}$ & $1.96666 \times 10^{-2}$ & $3.26875 \times 10^{-2}$ \\
\hline 1.0 & $1.302083 \times 10^{-2}$ & $3.0729166 \times 10^{-2}$ & $4.375 \times 10^{-2}$ \\
\hline
\end{tabular}

For $\mu=0.30$

$w(0)=\frac{p_{0} l^{4}}{24 E I}\left(\frac{5}{16}+\left(\frac{t}{l}\right)^{2}\left(\frac{1.3}{2 \times \frac{5.098}{6}}\right)\right)$

$w(0)=\frac{p_{0} l^{4}}{24 E I}\left(\frac{5}{16}+0.765\left(\frac{t}{l}\right)^{2}\right)$

$w(0)=\frac{p_{0} l^{4}}{384 E I}+3.1875 \times 10^{-2}\left(\frac{t}{l}\right)^{2} \frac{p_{0} l^{4}}{E I}$

$w(0)=w_{0}(f)+w_{0}(s)$

Table 2. Variation of maximum deflection with ratios of $t / l$ in Timoshenko beams with simply supported ends (case of uniformly distributed load over the entire beam span $l$ ), for $\mu=0.30$

\begin{tabular}{|c|c|c|c|}
\hline$t / l$ & $\begin{array}{c}\times \frac{p_{0} l^{4}}{E I} \\
w_{0}(f)\end{array}$ & $\begin{array}{c}\times \frac{p_{0} l^{4}}{E I} \\
w_{0}(s)\end{array}$ & $\begin{array}{c}\times \frac{p_{0} l^{4}}{E I} \\
w^{T}(0)\end{array}$ \\
\hline 0.005 & $1.302083 \times 10^{-2}$ & $7.96875 \times 10^{-7}$ & $1.3021626 \times 10^{-2}$ \\
\hline 0.01 & $1.302083 \times 10^{-2}$ & $3.1875 \times 10^{-6}$ & $1.3024017 \times 10^{-2}$ \\
\hline 0.02 & $1.302083 \times 10^{-2}$ & $1.275 \times 10^{-5}$ & $1.303358 \times 10^{-2}$ \\
\hline 0.05 & $1.302083 \times 10^{-2}$ & $7.96875 \times 10^{-5}$ & $1.3100517 \times 10^{-2}$ \\
\hline 0.08 & $1.302083 \times 10^{-2}$ & $2.04 \times 10^{-4}$ & $1.322483 \times 10^{-2}$ \\
\hline 0.10 & $1.302083 \times 10^{-2}$ & $3.1875 \times 10^{-4}$ & $1.333958 \times 10^{-2}$ \\
\hline 0.15 & $1.302083 \times 10^{-2}$ & $7.171875 \times 10^{-4}$ & $1.3738017 \times 10^{-2}$ \\
\hline 0.20 & $1.302083 \times 10^{-2}$ & $0.1275 \times 10^{-2}$ & $1.429583 \times 10^{-2}$ \\
\hline 0.40 & $1.302083 \times 10^{-2}$ & $0.51 \times 10^{-2}$ & $1.812083 \times 10^{-2}$ \\
\hline 0.60 & $1.302083 \times 10^{-2}$ & $1.1475 \times 10^{-2}$ & $2.449583 \times 10^{-2}$ \\
\hline 0.80 & $1.302083 \times 10^{-2}$ & $2.04 \times 10^{-2}$ & $3.342083 \times 10^{-2}$ \\
\hline 1.0 & $1.302083 \times 10^{-2}$ & $3.1875 \times 10^{-2}$ & $4.489583 \times 10^{-2}$ \\
\hline
\end{tabular}

\section{Illustrative problem}

A moderately thick rectangular cantilever beam of width $b$ and thickness $t$, with a span $l$ carries a point load $P$ at the free end $x=0$ and is fixed at the end $x=l$, as shown in Figure 2.

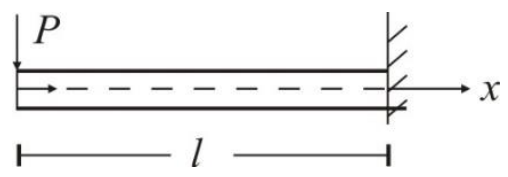

Figure 2. Moderately thick cantilever beam with a rectangular cross-section under point load

Considering an imaginary section $x$ from the fee end, the bending moment and shear force at the section is found from equilibrium considerations as

$M(x)=-P x$

$Q(x)=-P$

The differential equation of equilibrium is

$-E I \frac{d \alpha}{d x}=M(x)=-P x$

$E I \frac{d \alpha}{d x}=P x$

Integrating,

$\int E I \frac{d \alpha}{d x} d x=E I \alpha(x)=\int P x d x$

$E I \alpha(x)=\frac{P x^{2}}{2}+a_{1}$

where $a_{1}$ is an integration constant.

Using the boundary condition at the clamped end,

$\alpha(x=l)=0$

We have

$E I \alpha(x=l)=\frac{P l^{2}}{2}+a_{1}=0$

$\therefore a_{1}=-\frac{P l^{2}}{2}$

$E I \alpha(x)=\frac{P x^{2}}{2}-\frac{P l^{2}}{2}=\frac{P\left(x^{2}-l^{2}\right)}{2}$

$\alpha(x)=\frac{P\left(x^{2}-l^{2}\right)}{2 E I}$

This yields 
$\sigma_{x x}=\frac{P x z}{I}$

This result for $\sigma_{x x}$ is the same result obtained using the Euler-Bernoulli beam theory.

From the second differential equation of equilibrium,

$\frac{d}{d x}\left(\frac{d w}{d x}-\alpha(x)\right)+\frac{p(x)}{k G A}=0$

Integration yields

$\frac{d x}{d x}-\alpha(x)+\int \frac{p(x) d x}{k G A}=0$

$\frac{d w}{d x}-\alpha(x)+\frac{Q(x)}{k G A}=0$

$\frac{d w}{d x}-\alpha(x)=-\frac{Q(x)}{k G A}$

$\frac{d w}{d x}=-\frac{Q(x)}{k G A}+\alpha(x)=-\frac{P}{k G A}+\frac{P\left(x^{2}-l^{2}\right)}{2 E I}$

Integrating,

$\int \frac{d w}{d x} d x=\int d w=w(x)=\int\left\{\frac{-P}{k G A}+\frac{P\left(x^{2}-l^{2}\right)}{2 E I}\right\} d x$

$w(x)=\frac{-P x}{k G A}+\frac{P}{2 E I}\left(\frac{x^{3}}{3}-l^{2} x\right)+a_{2}$

where $a_{2}$ is the constant of integration.

Using the boundary conditions at the fixed end,

$w(x=l)=0$

We have

$w(x=l)=0=\frac{-P l}{k G A}+\frac{P}{2 E I}\left(\frac{l^{3}}{3}-l^{3}\right)+a_{2}$

$a_{2}=\frac{P l}{k G A}-\frac{P}{2 E I}\left(-\frac{2 l^{3}}{3}\right)$

$a_{2}=\frac{P l}{k G A}+\frac{2 P l^{3}}{6 E I}=\frac{P l}{k G A}+\frac{P l^{3}}{3 E I}$

Thus,

$w(x)=\frac{P}{2 E I}\left(\frac{x^{3}}{3}-l^{2} x\right)-\frac{P x}{k G A}+\frac{P l^{3}}{3 E I}+\frac{P l}{k G A}$

$w(x)=\frac{P}{E I}\left(\frac{x^{3}}{6}-\frac{l^{2}}{2} x+\frac{l^{3}}{3}\right)+\frac{P}{k G A}(l-x)$

$w(x)=w_{f}(x)+w_{s}(x)$
$w(0)=w(x=0)=\frac{P l^{3}}{3 E I}+\frac{P l}{k G A}$

$w(0)=\frac{P l^{3}}{3 E I}\left(1+\frac{3 E}{k G} \frac{I}{A l^{2}}\right)$

$w(0)=\frac{P l^{3}}{3 E I}\left(1+\frac{3 E}{k G} \frac{1}{12}\left(\frac{t}{l}\right)^{2}\right)$

$w(0)=\frac{P l^{3}}{3 E I}\left(1+\frac{E}{4 G k}\left(\frac{t}{l}\right)^{2}\right)$

From Equation (8),

$\frac{E}{G}=2(1+\mu)$

$w(0)=\frac{P l^{3}}{3 E I}\left(1+\frac{2(1+\mu)}{4 k}\left(\frac{t}{l}\right)^{2}\right)$

$w(0)=\frac{P l^{3}}{3 E I}\left(1+\frac{1+\mu}{2 k}\left(\frac{t}{l}\right)^{2}\right)$

For $\mu=0.25, k=\frac{5.08475}{6}$

$w(0)=\frac{P l^{3}}{3 E I}\left(1+\frac{1.25}{2 \times \frac{5.08475}{6}}\left(\frac{t}{l}\right)^{2}\right)$

$w(0)=\frac{P l^{3}}{3 E I}\left(1+0.7375\left(\frac{t}{l}\right)^{2}\right)$

$w(0)=w_{f}(0)+w_{s}(0)$

Table 3. Variation of maximum deflection with ratios of $t / l$ in cantilevered Timoshenko beams under point load $P$ at the free end (for $\mu=0.25$ )

\begin{tabular}{|c|c|c|c|}
\hline$t / l$ & $\begin{array}{l}\times \frac{P l^{3}}{E I} \\
w_{f}(0)\end{array}$ & $\begin{array}{l}\times \frac{P l^{3}}{E I} \\
w_{s}(0)\end{array}$ & $\begin{array}{l}\times \frac{P l^{3}}{E I} \\
w(0)\end{array}$ \\
\hline 0.005 & 0.333333 & $6.145833 \times 10^{-6}$ & 0.33333 \\
\hline 0.01 & 0.333333 & $2.45833 \times 10^{-5}$ & 0.333358 \\
\hline 0.02 & 0.333333 & $9.8333 \times 10^{-5}$ & 0.333432 \\
\hline 0.05 & 0.333333 & $6.14583 \times 10^{-4}$ & 0.333948 \\
\hline 0.08 & 0.333333 & $1.57333 \times 10^{-3}$ & 0.33491 \\
\hline 0.10 & 0.333333 & $2.45833 \times 10^{-3}$ & 0.335792 \\
\hline 0.15 & 0.333333 & $5.53125 \times 10^{-3}$ & 0.338865 \\
\hline 0.20 & 0.333333 & $9.8333 \times 10^{-3}$ & 0.343167 \\
\hline 0.40 & 0.333333 & 0.039333 & 0.372666 \\
\hline 0.60 & 0.333333 & 0.0885 & 0.421833 \\
\hline 0.80 & 0.333333 & 0.157333 & 0.490667 \\
\hline 1.0 & 0.333333 & 0.245833 & 0.5791666 \\
\hline
\end{tabular}


For $\mu=0.3, k=\frac{5.098}{6}$

$w(0)=\frac{P l^{3}}{3 E I}\left(1+\frac{1.3}{2 \times \frac{5.098}{6}}\left(\frac{t}{l}\right)^{2}\right)$

$w(0)=\frac{P l^{3}}{3 E I}\left(1+0.765\left(\frac{t}{l}\right)^{2}\right)$

Table 4. Variation of maximum deflection with ratios of $t / l$ in cantilevered Timoshenko beams under point load $P$ at the free end (for $\mu=0.30$ )

\begin{tabular}{|c|c|c|c|}
\hline$t / l$ & $\begin{array}{c}\times l^{3} \\
E I\end{array}$ & $\begin{array}{c}\times \frac{P l^{3}}{E I} \\
w_{f}(0)\end{array}$ & $\begin{array}{c}\times \frac{P l^{3}}{E I} \\
w(0)\end{array}$ \\
\hline 0.005 & 0.33333 & $6.375 \times 10^{-6}$ & 0.33334 \\
\hline 0.01 & 0.33333 & $2.55 \times 10^{-5}$ & 0.33336 \\
\hline 0.02 & 0.33333 & $1.02 \times 10^{-4}$ & 0.33344 \\
\hline 0.05 & 0.33333 & $6.375 \times 10^{-4}$ & 0.33397 \\
\hline 0.08 & 0.33333 & $1.632 \times 10^{-3}$ & 0.334965 \\
\hline 0.10 & 0.33333 & $2.55 \times 10^{-3}$ & 0.33588 \\
\hline 0.15 & 0.33333 & $5.7375 \times 10^{-3}$ & 0.339071 \\
\hline 0.20 & 0.33333 & 0.0102 & 0.34353 \\
\hline 0.40 & 0.33333 & 0.0408 & 0.374133 \\
\hline 0.60 & 0.33333 & 0.0918 & 0.425133 \\
\hline 0.80 & 0.33333 & 0.1632 & 0.49653 \\
\hline 1.0 & 0.33333 & 0.255 & 0.58833 \\
\hline
\end{tabular}

\section{DISCUSSION}

This work has successfully presented a variational formulation of the Timoshenko theory for the static flexural analysis of moderately thick beams with rectangular crosssections.

The formulation assumed a linear elastic, isotropic, homogenous beam material and accounted for the effect of transverse shear deformation. The displacement field components were assumed as Equations (15)-(17), and the kinematic relations for small displacement elasticity Equations (9-14) - used to obtained the strain fields as Equations (18), (20), (21), (22), (23), (24) and (25). Hooke's generalised stress-strain laws given as Equations (2)-(7) were used to obtain the stress fields as Equations (26)-(31). The internal stress resultants were obtained from the requirement of equilibrium of internal and external forces as Equations (37) and (40). The total potential energy functional $\Pi$ was obtained as the sum of the strain energy $U$ and the potential of the external load $V$ as Equation (54). The total potential energy functional $\Pi$ was observed to be a function of one independent variable, $x$, and two unknown functions $w(x)$ and $\alpha(x)$ and derivatives of the two unknown functions. The Euler-Lagrange conditions which are a system of two differential equations were used to obtain the differential equations of equilibrium of the Timoshenko beam under static flexure as a system of two coupled ordinary differential equations - Equations (64) and (70). The system of two coupled ordinary differential equations are expressed in decoupled form for homogeneous beams as Equations (78) and (84). Two specific illustrative cases of the closed form analytical solution of the Timoshenko beam equations were considered and solved. They were:

(i) moderately thick beam of rectangular cross-section simply supported at $x= \pm l / 2$ and subject to a uniformly distributed load of intensity $p_{0}$, and

(ii) moderately thick rectangular cantilever beam of span $l$ and beam $b$ and thickness $t$ with a point load $P$, applied at $x=0$, and fixed (clamped) at $x=l$.

Successive integration of the governing ODE was used to obtain the general solution for the unknown rotation $\alpha(x)$ in terms of three integration constants as Equation (101). The requirement that $\alpha(x)$ be an odd function, imposed by the symmetry of the problem was used to obtain two integration constants $c_{1}$ and $c_{3}$ as Equations (103) and (104). The third unknown integration constant $c_{2}$ was obtained using the boundary conditions at the simply supported ends as Equation (109). The solution for $\alpha(x)$ was thus fully determined as Equation (110). Equation (110) was used to obtain the differential equation for $w(x)$ as Equation (115). Integration of Equation (115) with respect to $x$ gave the general solution for $w(x)$ as Equation (117). Application of the boundary conditions Equations (118) and (119) gave the constant of integration $c_{4}$ as Equation (123), yielding the solution for $w(x)$ as Equation (125). The solution for $w(x)$ is observed to be expressed in terms of a flexural component $w_{f}(x)$ and a shear component $w_{s}(x)$. The maximum deflection was observed to occur at the center $x=0$, and was found as Equation (138). For $\mu=0.25$, the maximum deflection was obtained as Equation (141), which is decomposable into flexural and shear components. For $\mu=0.30$, the maximum deflection was obtained as Equation (147) which is also expressible as shear component and flexural components. In general, the expression for $w_{\max }$ is observed to depend on the ratio of the beam thickness to the beam $\operatorname{span} l$. Values of $w_{f}(0), w_{s}(0)$ and $w(0)$ for various values of $t / l$ ranging from $t / l=0.005$ to $t / l=1$ are tabulated for Poisson ratio values $\mu=0.25$ and $\mu=0.30$, and presented as Tables 1 and 2. Tables 1 and 2 show that for $t / l<0.02$ the contribution of shear to the overall deflection is insignificant being less than $0.1 \%$. At $t / l=0.1$ for $\mu=0.25$, the contribution of shear to the total deflection increases to $2.305 \%$, and to $46 \%$ for $t / l=0.60$ for $\mu=0.25$. For the moderately thick cantilever beam, the unknown rotation $\alpha(x)$ was obtained by integration and use of boundary conditions at the clamped end as Equation (158). The deflection $w(x)$ was obtained using Equation (158) and the Timoshenko beam equation as the ODE Equation (164). The general solution by integration was found as Equation (166). Application of boundary conditions at the fixed end yielded the unknown constant of integration as Equation (169). The solution for $w(x)$ was thus obtained as Equation (171), which is decomposable into flexural and shear components. The maximum deflection was observed to occur at the free end and is given by Equation (178). The maximum deflection was observed to depend upon the ratio $t / l$. For $\mu=0.25$, maximum deflection expression was found as Equation (180); while for $\mu=0.30$, the maximum deflection expression was obtained as Equation (182). Values of the maximum deflection for various values of the ratio $t / l$ for Poisson's ratio $\mu=0.25$, and $\mu=0.30$ are presented in tabular form as Tables 3 and 4 respectively for the moderately thick rectangular cantilever beam problem. 


\section{CONCLUSIONS}

The following conclusions are drawn from this study:

(i) the problem of flexure of moderately thick beams modelled and idealized using Timoshenko's first order shear deformation theory can be presented in variational form as a problem of finding the unknown displacements $w(x)$ and $\alpha(x)$ that minimize the total potential energy functional $\Pi$ or in differential form in terms of the system of two coupled ordinary differential equations obtained by use of the Euler-Lagrange conditions on the total potential energy functional.

(ii) the Timoshenko beam flexure problem can be formulated and solved using variational calculus methods.

(iii) mathematical solutions obtained show that the shear component of transverse deflections are expressed in terms of the square of the ratio of the beam thickness $t$ to the span $l$.

(iv) mathematical expressions obtained for the transverse deflection show the transverse deflection is decomposed into a shear component and a flexure component.

(v) the analytical expression obtained for the flexure component of the transverse deflection are exactly the same as obtained by the Euler-Bernoulli theory.

(vi) as the ratio of $t / l$ becomes very small, $t / l<0.02$ the contribution of the shear deformation to the resultant (overall) deflection is insignificant, being less than $0.1 \%$ and the solutions obtained become very close to the solutions obtained for the Euler-Bernoulli beam theory.

(vii) the contribution of shear deformation to the overall deflection becomes significant as $t / l>0.1$ and increases to $46 \%$ for $t / l=0.60$ for the case of simply supported moderately thick beam for $\mu=0.25$.

\section{REFERENCES}

[1] Ike CC, Ikwueze EU. (2018). Ritz method for the analysis of statically indeterminate Euler-Bernoulli beams. Saudi Journal of Engineering and Technology (SJEAT) 3(3): 133-140. https://doi.org/10.21276/sjeat20183.3.3

[2] Ike CC, Ikwueze EU. (2018). Fifth degree Hermittian polynomial shape functions for the finite element analysis of clamped simply supported Euler-Bernoulli beam. American Journal of Engineering Research (AJER) 7(4): 97-105.

[3] Ghugal YM, Dahake AG. (2013). Flexure of simply supported thick beams using refined shear deformation theory. World Academy of Science, Engineering and Technology, International Journal of Civil, Architectural, Structural and Construction Engineering 7(1): 82-91. https://doi.org/10.1999/1307-6892/9996869

[4] Nimbalkar VN, Dahake AG. (2015). Displacement and stresses for thick beam using new hyperbolic shear deformation theory. International Journal of Pure and Applied Research in Engineering and Technology 3(9): 120-130.

[5] Chavan VB, Dahake AG. (2015). A refined shear deformation theory for flexure of thick beam. International Journal of Pure and Applied Research in Engineering and Technology 3(9): 109-119.

[6] Pankade PM, Tupe DH, Salve SB. (2016). Static flexural analysis of thick isotropic beam using hyperbolic shear deformation theory. International Journal of Engineering Research 5(3): 565-571.

[7] Sayyad A. (2012). Static flexure and free vibration analysis of thick order shear deformation theories. International Journal of Applied Mathematics and Mechanics 8(14): 71-87.

[8] Ghugal Y. (2006). A two dimensional exact elasticity solution of thick beam. Departmental Report 1. Department of Applied Mechanics, Government Engineering College, Aurangabad India, pp 1-96.

[9] Timoshenko SP. (1921). On the correction for shear of differential equation for transverse vibrations of prismatic bars. Philosophical Magazine, Series 6 41: 742-746.

[10] Cowper GR. (1966). The shear coefficients in Timoshenko beam theory. ASME Journal of Applied Mechanics 33: 335-340.

[11] Ghugal YM, Sharma R. (2009). Hyperbolic shear deformation theory for flexure and vibration of thick isotropic beams. International Journal of Computational Methods 6(4): 585-604.

[12] Ghugal YM, Shimpi RP. (2002). A review of refined shear deformation theories for isotropic and anisotropic laminated beams. Journal of Reinforced Plastics and Composites 21: 775-813.

[13] Dahake AG, Ghugal YM. (2012). Flexure of thick simply supported beam using trigonometric shear deformation theory. International Journal of Scientific and Research Publications 2(11): 1-7. 10.29322

[14] Ghugal YM, Dahake AG. (2012). Flexure of thick beams using refined shear deformation theory. International Journal of Civil and Structural Engineering 3(2): 321335.

[15] Levinson M. (1981). A new rectangular beam theory. Journal of Sound and Vibration 74: 81-87. https://doi.org/10.1016/0022-460x(81)90493-4.

[16] Krishna Murty AV. (1984). Toward a consistent beam theory. AIAA Journal 22: 811-816.

[17] Baluch MH, Azad AK, Khidir MA. (1984). Technical theory of beams with normal strain. Journal of the Engineering Mechanics, Proceedings of the ASCE 110: 1233-1237.

[18] Bhimaraddi A, Chandreshekhara K. (1993). Observations on higher-order beam theory. Journal of Aerospace Engineering, Proceedings of ASCE, Technical Note 6: 403-413.

[19] Brickford WB. (1982). A consistent higher order beam theory. Development in Theoretical Applied Mechanics SECTAM 11: 137-150.

[20] Touratier M. (1991). An efficient standard plate theory. International Journal of Engineering Science 29(8): 901916.

[21] Vlasov VZ, Leontiev UN. (1996). Beams, plates and shells on elastic foundation. Chapter 1. pp. 1-8 (Translated from Russian by Barouch A. and Plez T.). Israel program for scientific translation Ltd, Jerusalem.

[22] Stein M. (1989). Vibration of beams and plate strips with three dimensional flexibility. Transaction ASME Journal of Applied Mechanics 56(1): 228-231.

[23] Kapdis P, Kalwane U, Salunkhe U, Dahake A. (2018). Flexural analysis of deep aluminium beam. Journal of Soft Computing in Civil Engineering 2(1): 71-84. https://doi.org/10.22115/SCCE.2018.49679

[24] Ghugal YM. (2006). A simple higher order theory for 
beam with transverse shear and transverse normal effect. Departmental Report No 4, Applied Mechanics Department, Government College of Engineering, Aurangabad, India, pp. 1-96.

\section{NOMENCLATURE}

$l$
$b$
$t$
$x, y, z$
$3 \mathrm{D}$
$\varphi(x)$
$\gamma_{x z}$
$\alpha(x)$
$x$
$w$
$\varepsilon_{x x}, \varepsilon_{y y}, \varepsilon_{z z}$
$\gamma_{x y}, \gamma_{y z}, \gamma_{x z}$
$\sigma_{x x}, \sigma_{y y}, \sigma_{z z}$
$\tau_{x y}, \tau_{y z}, \tau_{x z}$
$G$
$E$
$\mu$
$u, v, w$
$k$
$M(x)$
$Q(x)$
$U$
$V$
$\Pi$
$p(x)$

length of beam

width of beam

thickness of beam

three dimensional Cartesian coordinate

three dimensional (three dimensions)

rotation due to shear deformation

transverse shear angle

rotation due to bending deformation

longitudinal coordinate axis of beam

transverse deflection

normal strains

shear strains

normal stresses

shear stresses

shear modulus

Young's modulus of elasticity

Poisson's ratio

displacement field components in the $x, y$, and $z$ coordinate directions respectively

shear correction (modification) factor

bending moment

shear force

strain energy functional

potential of external load

total potential energy functional

applied load distribution
$A$
$I$
$F$

$\frac{\partial}{\partial w}$

$w^{\prime}(x)$

area of cross-section

moment of inertia

integrand in the total potential energy functional

partial derivative with respect to $w$

$a_{1} \quad a_{2}$

$\left.\begin{array}{cccc}c_{1} & c_{2} & c_{3} & c_{4}\end{array}\right\}$

derivative of $w(x)$ with respect to $x$

$p_{0}$

$p_{0}$
$P$

$\int$

$\iint$

constants of integration

\section{$\iiint$}

$\frac{d}{d x}$

\section{Subscripts}

$\begin{array}{ll}f & \text { flexural } \\ s & \text { shear } \\ b & \text { bending } \\ \max & \text { maximum }\end{array}$

\section{Superscripts}

c

corrected

$T$ Timoshenko

Abbreviations

EBT

$R^{3}$
Euler-Bernoulli beam theory

three dimensional region of integration 